\title{
Placental pathology in maternal autoimmune diseases-new insights and clinical implications
}

\author{
Ramya T. ${ }^{1 *}$, Umamaheswari G. ${ }^{2}$, Chaitra V. ${ }^{2}$
}

\begin{abstract}
${ }^{1}$ Department of Obstetrics and Gynecology, ${ }^{2}$ Department of Pathology, PSG Institute of Medical Sciences and Research, Coimbatore, Tamil Nadu, India
\end{abstract}

Received: 14 July 2017

Revised: 21 July 2017

Accepted: 08 August 2017

\section{*Correspondence:}

Dr. Ramya T.,

E-mail: ramya.t2003@gmail.com

Copyright: (C) the author(s), publisher and licensee Medip Academy. This is an open-access article distributed under the terms of the Creative Commons Attribution Non-Commercial License, which permits unrestricted non-commercial use, distribution, and reproduction in any medium, provided the original work is properly cited.

\begin{abstract}
Background: Pregnancy in women with autoimmune diseases is frequently associated with placental insufficiency leading to adverse perinatal outcome. Aim of the study was to investigate the presence and possible clinical significance of placental lesions in mothers with different autoimmune disorders.

Methods: In this retrospective study, 11 placentas from 10 mothers with diverse autoimmune diseases including systemic lupus Erythematosus (SLE), antiphospholipid antibodies (APLA), idiopathic thrombocytopenic purpura (ITP) and antinuclear antibodies (ANA) were studied.

Results: Placental correlates were reduced placental weight, maternal vascular under perfusion, abruption, villitis of unknown etiology, multifocal chorangiomatosis, distal villous immaturity, massive perivillous fibrin deposition/maternal floor infarction and foetal thrombotic vasculopathy. Of the 11 pregnancies 3 were untreated (1 SLE, 2 APLA) and resulted in intrauterine foetal demise. The lesions were more severe in these cases. All the treated pregnancies resulted in live born babies (8), of which 3 were growth restricted, 2 were complicated with oligohydramnios and 3 were delivered preterm.

Conclusions: In this group of diverse autoimmune disorders, placental lesions were not specific for each of them. Apart from maternal vascular under perfusion, lesions like villitis of unknown etiology, distal villous immaturity and massive perivillous fibrin deposition were identified and may recur in subsequent pregnancies and treatment should be directed towards modifying it. The placental examination should be mandatory in all cases of maternal autoimmunity and pregnancies with poor foetal outcome.
\end{abstract}

Keywords: Maternal autoimmune diseases, Maternal vascular under perfusion, Placental lesions

\section{INTRODUCTION}

Placenta plays a major role for a pregnancy to proceed normally, ensuring an adequate blood supply to support and promote the growth of the developing foetus. Autoimmune disease, both organ specific and non-organ specific is associated with intrauterine growth retardation (IUGR), preterm delivery and intrauterine foetal demise (IUFD) and appears to be a consequence of failure of the normal uterine physiologic changes to occur with resultant placental insufficiency and the features that accompany a failing placenta. ${ }^{1}$ Despite being an important etiology of poor perinatal outcome, placental lesions and its clinical correlation of autoimmune diseases lack a unified diagnostic criterion.

These clinical associations as well as foetal outcome suggest that there may be multiple pathologies that 
complicate and disrupt pregnancy in patients with autoimmune disorders. ${ }^{2}$

Given these uncertainties surrounding the placental pathology of autoimmune diseases, we sought to determine the presence and possible clinical significance of maternal vascular lesions, chronic villitis of unknown etiology (VUE), distal villous immaturity (DVI), massive perivillous fibrin deposition/maternal floor infarction (MPVFD/MFI) in placentas of mothers with different autoimmune disorders.

\section{METHODS}

This retrospective study was carried out from January 2014 to April 2017 in the Departments of Obstetrics and Gynecology and Department of Pathology at PSG IMS and R.

A total of 11 placentas were studied from ten mothers with maternal immunological disorders which included systemic lupus erythematosus (SLE) with secondary anti phospholipid antibodies (APLA), primary anti phospholipid antibodies (APLA), idiopathic thrombocytopenic purpura (ITP) and one with antinuclear antibody (ANA).

All patients had singleton pregnancies without any foetal malformations. Infants below tenth percentile were considered as having intrauterine growth restriction.

\section{According to the revised sydney classification criteria, apla is defined by}

- Presence of lupus anticoagulant (LA) in plasma on two or more occasions at least 12 weeks apart

- Presence of moderate to high levels of anticardiolipin (aCL) (IgG or $\operatorname{IgM}$ ) in serum or plasma (i.e., >40 IgG phospholipid units (GPL)/mL or $\operatorname{IgM}$ phospholipid units (MPL)/mL or $>99$ th percentile) on two or more occasions at least 12 weeks apart

- Presence of moderate to high levels of anti-beta-2 glycoprotein I antibodies (IgG or IgM) in serum or plasma $\left(>99^{\text {th }}\right.$ percentile) on two or more occasions at least 12 weeks apart. ${ }^{2}$

Patients with SLE were diagnosed according to the American Rheumatism Association Diagnostic Criteria. ${ }^{2}$ Both patients with ITP were diagnosed during the prepregnancy period. ${ }^{4}$

An entire placenta was available from all 10 patients and were fixed in $10 \%$ formalin. Placentas were weighed after the membranes and umbilical cord were trimmed off and compared with the reference weights for trimmed singleton placentas. Two rolls of membranes were taken and umbilical cord was examined for length, diameter, colour, insertion site and number of twists. ${ }^{5}$ Photography of the placentas were routinely taken after examining foetal and maternal surfaces.
The placentas were cut at $1 \mathrm{~cm}$ intervals and the parenchyma was assessed for colour, sponginess and specific lesions. Relevant lesional sections were taken along with membrane roll, cord and normal parenchyma. All the histologic sections were stained with hematoxylin and eosin.

\section{RESULTS}

Over a period of 3-years 11 placentas from 10 mothers with the clinical and laboratory evidence of maternal autoimmune diseases were included in the study.

Two out of ten studied patients had SLE with secondary APLA, five had primary APLA, two had ITP and one with ANA positivity. One patient with ITP had two consecutive pregnancies and both placentas were studied

\section{Patients with SLE}

Clinical data and placental findings from the two studied patients with SLE are shown in Table 1. Both of our patients had SLE with secondary APLA.

Patient 1 was diagnosed 9 years before the index pregnancy. Her first pregnancy was an induced abortion owing to SLE flare, while the second was a spontaneous abortion. She had active renal involvement and superimposed preeclampsia in the present pregnancy. She was treated with low molecular weight heparin (LMWH), immunomodulators, anti-hypertensives and aspirin. She delivered a preterm small for gestational age (SGA) baby at 34 weeks of gestation due to preterm premature rupture of membranes (PPROM). Her placenta was small with thin hypercoiled cord ( 8 twists for $11.0 \mathrm{cms}$ of cord length-). Microscopically it showed maternal vascular underperfusion which included distal villous hypoplasia, increased syncytial knots, abundant perivillous fibrin deposition and calcification. Multifocal chorangiomatosis (Figure 1) was also noted.

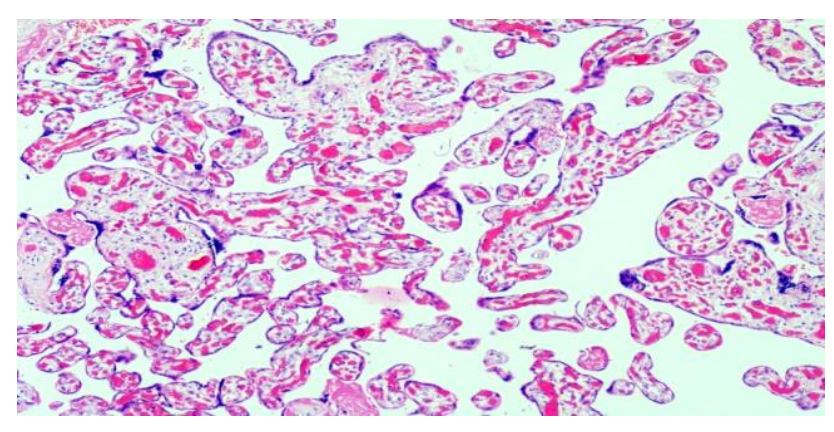

Figure 1: Multifocal chorangiomatosis-immature intermediate villi with proliferating capillaries and surrounding pericytes ( $\mathrm{H}$ and $\mathrm{E}, 4 \mathrm{X})$.

Patient 2 was a second gravida with previous spontaneous abortion at 8 weeks. Now she was referred with an intrauterine foetal demise at 24 weeks of gestation. On evaluation she was found to have malar rash, 
thrombocytopenia and presence of anti Sm antibodies. On investigating further, she also had an abnormal lupus anticoagulant (LA), with elevated titres of anticardiolipin (aCL) antibody $\mathrm{IgG}$ and beta 2 glycoprotein $1 \mathrm{IgM}$.

The placenta was very small (less than $10^{\text {th }}$ percentile) and had thin, flat umbilical cord. The parenchyma grossly showed marginal atrophy and a basal firm yellow nodule.
Microscopically, some of the chorionic plate and stem villous vessels showed recanalizing thrombi and haemorrhagic endovasculitis (foetal thrombotic vasculopathy). There was laminar necrosis of the decidua, patchy lymphohistiocytic villitis, intervillositis (Figure 2), microinfarcts and foci of avascular, hyalinized villi. Basal haematoma with indentation of parenchyma was also noted.

Table 1: Patients with SLE.

\begin{tabular}{|c|c|c|}
\hline Details & Patient-1 & Patient-2 \\
\hline Maternal age & 27 & 21 \\
\hline autoimmune etiology & $\begin{array}{l}\text { SLE (Class } 4 \text { lupus nephritis), } \\
\text { secondary APLA, hypothyroidism }\end{array}$ & SLE with secondary APLA \\
\hline Duration since diagnosis & 9 years & Post -partum \\
\hline Laboratory data & $\begin{array}{l}\text { aCL IgM, IgG positive, anti-ds DNA } \\
\text { positive }\end{array}$ & $\begin{array}{l}\text { ANA positive (Sm positive, nucleosome } \\
\text { positive), Abnormal LA, aCL IgG positive, anti- } \\
\text { beta } 2 \text { glycoprotein } 1 \text { IgM positive, } \\
\text { Thrombocytopenia }\end{array}$ \\
\hline $\begin{array}{l}\text { Previous obstetric } \\
\text { history }\end{array}$ & $\begin{array}{l}\text { G1-MTP at } 8 \text { weeks due to SLE flare } \\
\text { G2-spontaneous abortion at } 18 \text { weeks } \\
\text { G3-PP }\end{array}$ & $\begin{array}{l}\text { G1-Spontaneous abortion at } 8 \text { weeks } \\
\text { G2-PP }\end{array}$ \\
\hline $\begin{array}{l}\text { Gestational age at } \\
\text { delivery }\end{array}$ & $34 \mathrm{~W}+1 \mathrm{D}$ & $24 W+1 D$ \\
\hline $\begin{array}{l}\text { Course during } \\
\text { pregnancy/complications }\end{array}$ & $\begin{array}{l}\text { Superimposed preeclampsia, IUGR, } \\
\text { PPROM }\end{array}$ & Malar rash \\
\hline $\begin{array}{l}\text { Treatment during } \\
\text { pregnancy }\end{array}$ & $\begin{array}{l}\text { LMWH, antihypertensives, Aspirin, } \\
\text { Steroids, immunomodulators and } \\
\text { eltroxin }\end{array}$ & None \\
\hline Foetal outcome & SGA, Live birth & IUFD \\
\hline Foetal weight & $1.4 \mathrm{~kg}$ & $150 \mathrm{gms}$ \\
\hline \multicolumn{3}{|l|}{ Gross } \\
\hline Placental weight & $* 194.0 \mathrm{gms}\left(<10^{\text {th }}\right.$ percentile $)$ & $\begin{array}{l}* 19.0 \mathrm{gms}\left(<10^{\text {th }} \text { percentile }\right) \\
* \text { Thin, flat cord }\end{array}$ \\
\hline Cord & *Thin, hypercoiled cord & $\begin{array}{l}\text { Basal firm yellow nodule, at the level of cord } \\
\text { insertion }\end{array}$ \\
\hline Any specific lesions & *Small subchorionic haemorrhage & Marginal atrophy and basal haematoma \\
\hline \multirow[t]{2}{*}{ Microscopy } & $\begin{array}{l}\text { Maternal vascular underperfusion } \\
\text { (Distal villous hypoplasia, Increased } \\
\text { syncytial knots, Abundant perivillous } \\
\text { fibrin deposition and calcification) }\end{array}$ & $\begin{array}{l}\text { Thrombi, infarcts } \\
\text { Recanalizing thrombi and haemorrhagic } \\
\text { endovasculitis of stem villous vessels, avascular } \\
\text { hyalinized villi (Foetal thrombotic vasculopathy) }\end{array}$ \\
\hline & Multifocal chorangiomatosis & $\begin{array}{l}\text { Retroplacental haematoma } \\
\text { Villitis and perivillositis } \\
\text { Laminar necrosis of the decidua }\end{array}$ \\
\hline
\end{tabular}

\section{Patients with APLA}

Clinical data and placental findings from the five studied patients with primary antiphospholipid antibodies are shown in Table 2 . In patients 4 and 6 , presence of APLA were diagnosed postpartum and the pregnancies resulted in intrauterine foetal demise. In the remaining three patients two were diagnosed even before attempting the studied pregnancies and were treated optimally which resulted in live births. Patient 3 was diagnosed to have
APLA at the end of first trimester. She was tested during the ongoing pregnancy as she had previously delivered a premature, growth restricted baby with absent diastolic flow in umbilical artery doppler. Two out of the 3 live births were appropriate for gestational age (AGA). Of the three treated patients two were delivered near term, while the third (patient 3) was preterm.

In four out of 5 patients, the placental weight was small for gestational age. The placenta of the three live born 
fetuses showed maternal vascular under perfusion (infarcts, intervillous thrombi and decidual vasculopathy) and vascular ectasia with hypercapillarization.

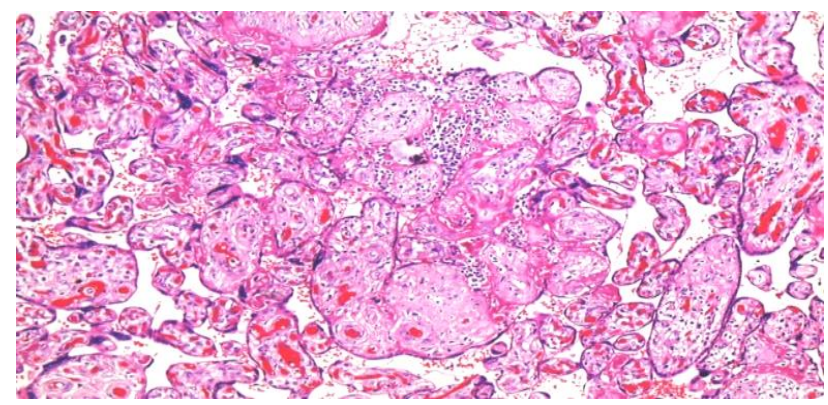

Figure 2: Lymphohistiocytic infiltrate in VUE (H and E, 10X).

Placenta of patient 6 (IUFD) showed retroplacental haematoma with parenchymal indentation, subchorionic haematoma and intervillous thrombus. Interestingly the other IUFD (patient 4) had a AGA stillbirth and her placenta showed distal villous immaturity (Figure 3).

There were patchy, multifocal chorangiomatosis and villous stromal karyorrhexis. Lowgrade, focal villitis of unknown etiology was also noted.

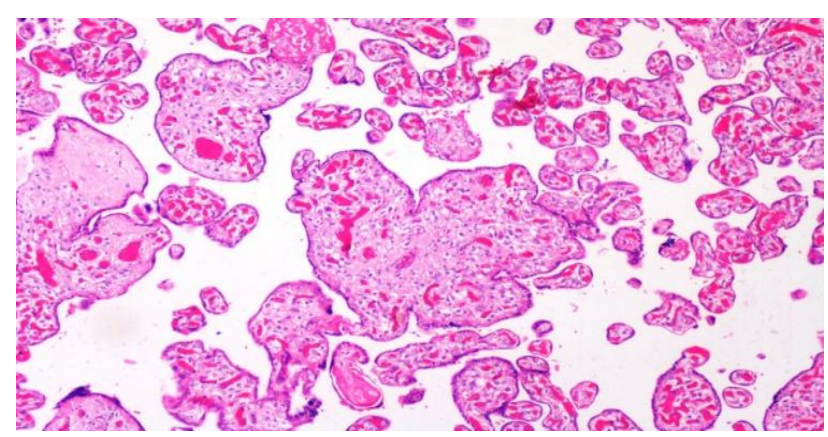

Figure 3: Distal villous immaturity- immature villi with irregular outlines, paucity of vasculosyncytial membranes (H and E, 10X).

\section{Patients with ITP}

Clinical data and placental findings from the 3 studied pregnancies with idiopathic thrombocytopenic purpura are shown in Table 3.

Patient 9 had two successive pregnancies that were studied. Both the pregnancies were complicated by oligohydramnios. She had active disease during her first pregnancy and was treated with immunoglobulins, steroids and multiple transfusions. None of the babies were SGA or stillborn.

Almost all the placentas were $\leq 10^{\text {th }}$ percentile. Maternal vascular underperfusion was found in 2 placentas of the three studied pregnancies (patient 8 and 9B) with ITP.
Hypercoiling of the cord and haemorrhage around the umbilical vessels were present in patient 9B.

Transmural massive perivillous fibrin deposition (maternal floor infarction) was noted in patient 9A (Figure 4). Intervillous thrombi and lymphocytic deciduitis were also identified.

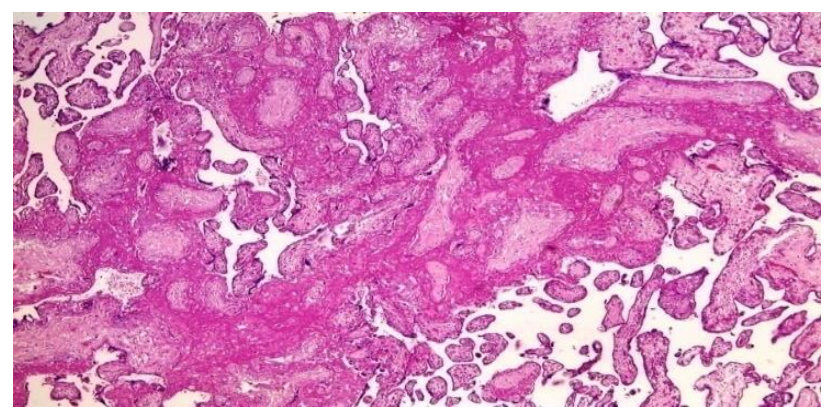

Figure 4: Massive perivillous fibrin depositionmarked increase in transmural perivillous fibrin deposition involving $>50 \%$ of the villi.

\section{Patient with ANA}

Clinical data and placental findings of the patient with antinuclear antibody is shown in Table 3.

Patient 10 was a low risk primigravida and on routine screening was found to have high resistance flow in uterine artery at mid trimester. On screening for autoimmunity she tested positive for antinuclear antibodies. She was treated with LMWH from 20 weeks and delivered a SGA baby at near term.

Placenta showed both maternal vascular under perfusion and foetal thrombotic vasculopathy which was evident by infarcts, distal villous hypoplasia, hypercapillarization, increased syncytial knots and fibrin thrombi of chorionic plate and stem villous vessels with large foci of avascular hyalinized villi (more than two foci/average of 15 or more affected villi/slide/ foetal vessel lesions) (Figure 5). ${ }^{6,7}$

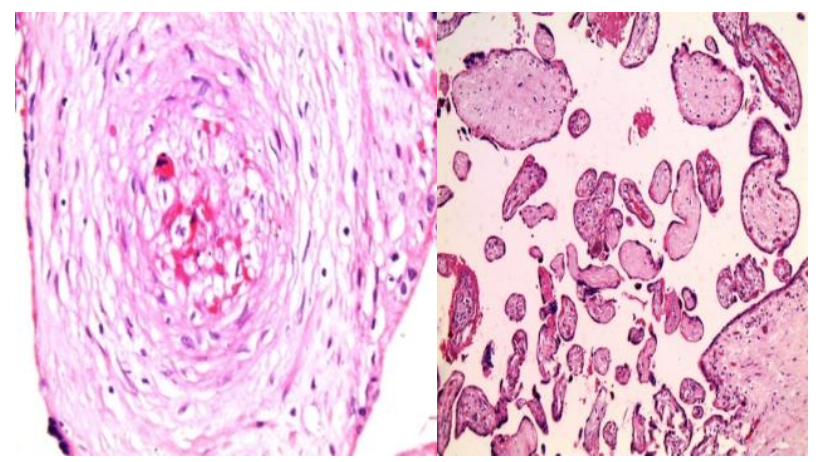

Figure 5: Foetal thrombotic vasculopathy-A) Stem villi with recanalizing thrombus B) groups of avascular hyalinized villi, $>15$ villi. 
Table 2: Patients with antiphospholipid antibodies.

\begin{tabular}{|c|c|c|c|c|c|}
\hline Details & Patient 3 & Patient 4 & Patient 5 & Patient 6 & Patient 7 \\
\hline Maternal age & 24 Years & 23 years & 19years & 22years & 28 years \\
\hline $\begin{array}{l}\text { Autoimmune } \\
\text { etiology }\end{array}$ & APLA & $\begin{array}{l}\text { APLA, Overt } \\
\text { hypothyroidism }\end{array}$ & APLA & APLA & $\begin{array}{l}\text { APLA, old } \\
\text { cerebral venous } \\
\text { thrombosis }\end{array}$ \\
\hline $\begin{array}{l}\text { Duration since } \\
\text { diagnosis }\end{array}$ & First trimester of PP & $\begin{array}{l}\text { Post-partum of } \\
\text { present pregnancy }\end{array}$ & 1 year (post abortal) & $\begin{array}{l}\text { Post-partum } \\
\text { (present } \\
\text { pregnancy) }\end{array}$ & 9 years \\
\hline Laboratory data & aCL IgM positive & $\begin{array}{l}\text { Anti-beta } 2 \\
\text { glycoprotein } 1 \\
\text { IgG positive }\end{array}$ & aCL IgM positive & $\begin{array}{l}\text { Abnormal LA, } \\
\text { aCL IgG } \\
\text { Positive, anti } \\
\text { beta } 2 \\
\text { glycoprotein } 1 \\
\text { IgG and IgM } \\
\text { positive }\end{array}$ & $\begin{array}{l}\text { Abnormal LA, } \\
\text { aCL IgG positive }\end{array}$ \\
\hline $\begin{array}{l}\text { Previous obstetric } \\
\text { history }\end{array}$ & $\begin{array}{l}\text { G1-emergency LSCS at } \\
35 \text { weeks (IUGR, AEDF) } \\
\text { G2-MTP at } 8 \text { weeks } \\
\text { G3-PP }\end{array}$ & $\begin{array}{l}\text { G1-Spontaneous } \\
\text { abortion at } \\
\text { 20weeks } \\
\text { G2-PP }\end{array}$ & $\begin{array}{l}\text { G1-missed abortion } \\
\text { G2-missed abortion } \\
\text { G3-PP }\end{array}$ & Primi & $\begin{array}{l}\text { G1-normal } \\
\text { vaginal } \\
\text { delivery(treated) }\end{array}$ \\
\hline $\begin{array}{l}\text { Gestational age at } \\
\text { delivery }\end{array}$ & $33 \mathrm{~W}+2 \mathrm{D}$ & $38 \mathrm{~W}+5 \mathrm{D}$ & 36weeks & $32 W+6 D$ & $37 \mathrm{~W}+3 \mathrm{D}$ \\
\hline $\begin{array}{l}\text { Course during } \\
\text { pregnancy/ } \\
\text { complications }\end{array}$ & $\begin{array}{l}\text { Subclinical } \\
\text { hypothyroidism, GGI }\end{array}$ & $\begin{array}{l}\text { Cervical } \\
\text { encirclage done at } \\
13 \text { weeks (history } \\
\text { indicated) }\end{array}$ & GGI & $\begin{array}{l}\text { Grade } 3 \\
\text { abruption }\end{array}$ & None \\
\hline $\begin{array}{l}\text { Treatment during } \\
\text { pregnancy }\end{array}$ & $\begin{array}{l}\text { Aspirin } 75 \mathrm{mg} \text { from } 10 \\
\text { weeks UFH from } 12 \\
\text { weeks, steroids for lung } \\
\text { maturity }\end{array}$ & Eltroxin & $\begin{array}{l}\text { LMWH from } 12 \\
\text { weeks }\end{array}$ & None & $\begin{array}{l}\text { LMWH from } 12 \\
\text { weeks }\end{array}$ \\
\hline Foetal outcome & AGA, Live birth & IUFD & AGA, Live birth & IUFD & SGA, Live birth \\
\hline Foetal weight & $1.8 \mathrm{~kg}$ & $3.4 \mathrm{KG}$ & $2.75 \mathrm{~kg}$ & $1.7 \mathrm{~kg}$ & $2.44 \mathrm{~kg}$ \\
\hline \multicolumn{6}{|l|}{ Gross } \\
\hline Placental weight & $307.0 \mathrm{gms}$ & $\begin{array}{l}389.0 \text { gms }\left(<10^{\text {th }}\right. \\
\text { percentile }\end{array}$ & $445.0 \mathrm{gms}$ & $\begin{array}{l}271.0 \mathrm{gms} \\
\left(<10^{\text {th }}\right. \\
\text { percentile })\end{array}$ & $\begin{array}{l}345.0 \mathrm{gms}\left(<10^{\text {th }}\right. \\
\text { percentile })\end{array}$ \\
\hline Cord & $\begin{array}{l}\text { Marginally inserted } \\
\text { hypercoiled cord }\end{array}$ & $\begin{array}{l}\text { Edematous } \\
\text { hypocoiled cord }\end{array}$ & Eccentrically placed & $\begin{array}{l}\text { Hypocoiled } \\
\text { cord }\end{array}$ & $\begin{array}{l}\text { Eccentrically } \\
\text { placed }\end{array}$ \\
\hline \multirow[t]{2}{*}{ Any specific lesions } & $\begin{array}{l}\text { Basal yellow brown } \\
\text { nodule, } 1.0 \mathrm{~cm} \text { from cord } \\
\text { insertion }\end{array}$ & None & None & $\begin{array}{l}\text { Retroplacental } \\
\text { haematoma } \\
\text { with } \\
\text { parenchymal } \\
\text { indentation }\end{array}$ & None \\
\hline & & & & $\begin{array}{l}\text { Subchorionic } \\
\text { haematoma } \\
\text { Intervillous } \\
\text { thrombus }\end{array}$ & \\
\hline \multirow[t]{4}{*}{ Microscopy } & Intervillous thrombi & $\begin{array}{l}\text { Distal villous } \\
\text { immaturity }\end{array}$ & $\begin{array}{l}\text { Vascular ectasia of } \\
\text { chorionic plate, stem } \\
\text { villous vessels and } \\
\text { hypercapillarisation } \\
\text { of distal villi }\end{array}$ & $\begin{array}{l}\text { Retroplacental } \\
\text { and } \\
\text { subchorionic } \\
\text { haematoma, } \\
\text { intervillos } \\
\text { thrombus, } \\
\text { C/W abruption }\end{array}$ & $\begin{array}{l}\text { Fibrin thrombi in } \\
\text { the decidual } \\
\text { Vessels } \\
\text { and focal laminar } \\
\text { necrosis }\end{array}$ \\
\hline & Infarct & $\begin{array}{l}\text { Patchy } \\
\text { chorangiomatosis }\end{array}$ & $\begin{array}{l}\text { Distal villous } \\
\text { immaturity }\end{array}$ & & $\begin{array}{l}\text { Distal villous } \\
\text { immaturity }\end{array}$ \\
\hline & $\begin{array}{l}\text { Laminar necrosis, } \\
\text { decidua }\end{array}$ & $\begin{array}{l}\text { Lowgrade, focal } \\
\text { villitis of } \\
\text { unknown } \\
\text { aetiology }\end{array}$ & & & $\begin{array}{l}\text { Maternal } \\
\text { vascular } \\
\text { underperfusion }\end{array}$ \\
\hline & $\begin{array}{l}\text { Maternal vascular } \\
\text { underperfusion }\end{array}$ & & & & \\
\hline
\end{tabular}


Table 3: Patients with idiopathic thrombocytopenic purpura and other diseases.

\begin{tabular}{|c|c|c|c|c|}
\hline Details & Patient 8 & Patient 9A & Patient 9B & Patient 10 \\
\hline Maternal age & 26 years & 29 years & 31 years & 27 years \\
\hline autoimmune etiology & ITP & ITP & ITP & ANA positive \\
\hline Duration since diagnosis & 2 years & 14 years & 16 years & 20 weeks of gestation \\
\hline Laboratory data & $\begin{array}{l}\text { Severe } \\
\text { Thrombocytopenia }\end{array}$ & $\begin{array}{l}\text { Severe } \\
\text { Thrombocytopenia, } \\
\text { ANA-Anticytoplasmic } \\
\text { antibodies positive }\end{array}$ & $\begin{array}{l}\text { Severe } \\
\text { Thrombocytopenia, } \\
\text { ANA- } \\
\text { Anticytoplasmic } \\
\text { antibodies positive }\end{array}$ & $\begin{array}{l}\text { ANA-Anticytoplasmic } \\
\text { antibodies positive }\end{array}$ \\
\hline $\begin{array}{l}\text { Previous obstetric } \\
\text { history }\end{array}$ & $\begin{array}{l}\text { G1-normal vaginal } \\
\text { delivery } \\
\text { G2-PP }\end{array}$ & Primi & $\begin{array}{l}\text { G1- normal vaginal } \\
\text { delivery, } \\
\text { postpartum } \\
\text { splenectomy } \\
\text { G2-PP }\end{array}$ & Primi \\
\hline $\begin{array}{l}\text { Gestational age at } \\
\text { delivery }\end{array}$ & $38 W+6 D$ & $36 \mathrm{~W}+6 \mathrm{D}$ & $38 \mathrm{~W}+4 \mathrm{D}$ & $37 W+4 D$ \\
\hline $\begin{array}{l}\text { Course during } \\
\text { pregnancy/complications }\end{array}$ & - & $\begin{array}{l}\text { PPROM, } \\
\text { Oligohydramnio, } \\
\text { Postpartum splenectomy }\end{array}$ & Oligohydramnios & Subclinical hypothyroidism \\
\hline $\begin{array}{l}\text { Treatment during } \\
\text { pregnancy }\end{array}$ & Platelet transfusions & $\begin{array}{l}\text { IV Ig, steroids, platelet } \\
\text { transfusions }\end{array}$ & $\begin{array}{l}\text { Steroids, platelet } \\
\text { transfusions }\end{array}$ & LMWH from 20 weeks \\
\hline Foetal outcome & AGA, live born & AGA, liveborn & AGA, liveborn & SGA, liveborn \\
\hline Foetal weight & $2.6 \mathrm{~kg}$ & $2.33 \mathrm{~kg}$ & $3.05 \mathrm{~kg}$ & $2.23 \mathrm{~kg}$ \\
\hline \multicolumn{5}{|l|}{ Gross } \\
\hline Placental weight & $\begin{array}{l}312.0 \mathrm{gms}\left(<10^{\text {th }}\right. \\
\text { percentile })\end{array}$ & $\begin{array}{l}358.0 \mathrm{gms}\left(<10^{\text {th }}\right. \\
\text { percentile })\end{array}$ & $\begin{array}{l}410.0 \mathrm{gms}\left(=10^{\text {th }}\right. \\
\text { percentile })\end{array}$ & 386.0gms $\left(<10^{\text {th }}\right.$ percentile $)$ \\
\hline Cord & $\begin{array}{l}\text { Eccentrically } \\
\text { placed, edemato us } \\
\text { cord }\end{array}$ & $\begin{array}{l}\text { Eccentrically placed, flat, } \\
\text { hypocoiled cord }\end{array}$ & $\begin{array}{l}\text { Hypercoiled, } \\
\text { edematous cord }\end{array}$ & Hypocoiled cord \\
\hline Any specific lesions & $\begin{array}{l}2 \text { full thickness firm } \\
\text { yellow lesions with } \\
\text { central } \\
\text { haemorrhagic area }\end{array}$ & $\begin{array}{l}\text { Three basal wedge } \\
\text { shaped, pale brown, firm } \\
\text { areas }\end{array}$ & None & $\begin{array}{l}\text { Three basal to full thickness, } \\
\text { firm yellow brown lesions }\end{array}$ \\
\hline \multirow[t]{5}{*}{ Microscopy } & Intervillous thrombi & $\begin{array}{l}\text { Massive perivillous fibrin } \\
\text { deposition(transmural) }\end{array}$ & $\begin{array}{l}\text { Haemorrhage } \\
\text { around the three } \\
\text { umbilical vessels }\end{array}$ & Decidual laminar necrosis \\
\hline & Infarcts & Intervillous thrombi & $\begin{array}{l}\text { Decidual laminar } \\
\text { necrosis }\end{array}$ & Infarcts \\
\hline & $\begin{array}{l}\text { DVH, } \\
\text { hypercapillarization, } \\
\text { increased syncytial } \\
\text { knots, C/W } \\
\text { maternal vascular } \\
\text { underperfusion }\end{array}$ & Lymphocytic deciduitis & $\begin{array}{l}\text { Incipient } \\
\text { chorangiosis }\end{array}$ & $\begin{array}{l}\text { Chorionic plate and stem } \\
\text { villous vessels show fibrin } \\
\text { thrombi }\end{array}$ \\
\hline & & & $\begin{array}{l}\text { Maternal vascular } \\
\text { underperfusion }\end{array}$ & $\begin{array}{l}\text { Large Foci of avascular } \\
\text { hyalinized villi }\end{array}$ \\
\hline & & & & $\begin{array}{l}\text { DVH, hypercapillarization, } \\
\text { increased syncytial knots, } \\
\text { C/W maternal vascular } \\
\text { underperfusion and foetal } \\
\text { vascular obstruction }\end{array}$ \\
\hline
\end{tabular}

\section{DISCUSSION}

Many physiologiocal changes must occur to allow the foetus to persist and grow. Part of the implantation process involves syncytiotrophoblast invasion and erosion of maternal endometrial blood vessels resulting in the development of a utero-placental circulation. Ultimately, the trophoblast becomes incorporated into the wall of the vessel which has now become a thin walled, dilated vascular channel with lack of muscle, thereby ensuring an adequate blood supply to the developing foetus. These vascular changes have been termed physiological change and lack of physiological change or decidual vasculopathy has been found to be a significant placental feature in certain diseases, for example preeclampsia alone, chronic hypertension with 
superimposed preeclampsia, autoimmune diseases and idiopathic SGA infants. ${ }^{8-10}$

In this report, we describe clinical and pathologic findings of 11 placentas from 10 patients with autoimmune diseases. We prefer to specify the lesions that are present rather than to embrace them all in the imprecise terminology of placental insufficiency. The primary pathology observed in the present study was maternal vascular underperfusion (fibrin thrombi in the decidual vessels, intervillous thrombi, infarcts, distal villous hypoplasia and increased syncytial knotting). ${ }^{8-10}$ Interestingly, the other pathological features observed were chorangiomatosis, villitis, massive perivillous fibrin deposition, distal villous immaturity, abruption and foetal thrombotic vasculopathy.

Distal villous immaturity (DVI) is a maturation defect of the terminal villi. ${ }^{13}$ Microscopically the terminal villi are enlarged with excessive stroma, increased vascularity, with a continuous layer of syncytiotrophoblast, paucity of vasculosyncytial membranes and lack peripheral polarization of capillaries. This in turn decreases the efficiency of maternal-foetal exchange leading to late gestational hypoxia and IUFD. This finding is most often associated with maternal diabetes. DVI was apparent in the only term IUFD of our study. Contrary to the observations made so far in the literature, there was no evidence of glucose intolerance in the mother, but she had primary APLA. Presence of DVI in autoimmune diseases remains speculative and needs further characterization.

Chorangiomatosis involves immature intermediate villi or stem villi and the vessels are surrounded by pericytes with increased stromal cellularity and collagen. Diffuse multifocal chorangiomatosis is associated with extreme prematurity, IUGR, preeclampsia and it has been suggested that it may be a developmental abnormality of the villi. ${ }^{14}$ This was the major lesion along with maternal vascular underperfusion in one of the studied SLE patients with secondary APLA. The pregnancy was complicated by preeclampsia, prematurity and intrauterine growth restriction.

The other patient who had SLE with secondary APLA was diagnosed postpartum and was not treated antenatally. She had a severe growth retarded foetus which resulted in stillbirth. The predominant lesion in the placenta was villitis of unknown etiology (VUE), perivillositis and retroplacental haematoma. ${ }^{15}$ The villi were invaded and expanded by lymphohistiocytic infiltrate and showed secondary changes of ischemia,infarction,recanalizing thrombi in the stem villous vessels with resultant groups of avascular villi (foetal thrombotic vasculopathy).

VUE is a well-recognized entity, but remains a significant challenge because of its frequency, high recurrence rate and associated poor outcome. The most accepted theory is that VUE is an immune reaction akin to placental "rejection". ${ }^{16}$

Thrombi in the foetal circulation of the placenta cause a pattern of clustered avascular fibrotic villi called foetal thrombotic vasculopathy (FTV). Causal factors of FTV include stasis, maternal autoimmune diseases and vascular injury. The most serious outcomes include growth restriction, neurologic injury and perinatal death. 6,7

Our patient with ANA positivity also had foetal thrombotic vasculopathy with superimposed maternal vascular under perfusion and resultant SGA baby. ${ }^{11,12}$

Apart from maternal vascular underperfusion, one of the placenta of ITP patient also showed massive perivillous fibrin deposition/maternal floor infarction (MPVFD/MFI). This particular patient had active disease during pregnancy and was treated with immunoglobulins, steroids and had improved pregnancy outcome. MPVFD/MFI has a recurrence rate of $30 \%$ or more. ${ }^{17} \mathrm{We}$ had an opportunity to study the subsequent pregnancy of the same patient where the lesion did not recur. There were no associations found between placental lesions and foetal outcome in all three studied ITP cases.

\section{CONCLUSION}

Placenta remains an undervalued and underutilized surgical specimen in the evaluation of maternal autoimmune disorders. Studies have clearly demonstrated that there are a multitude of mechanisms where by autoimmunity can prevent normal placental development and can cause significant pathology. In this group of diverse autoimmune diseases placental lesions were not specific for any of them.

But it appears that diseases that are clinically more severe have more severe placental vascular changes, related to foetal outcome.

Therefore, although the histopathologic lesions are similar, there appears to be a "grading" in severity. Placental thrombosis and infarction are just one part of the placental lesions. Analyzing previous placentas has been helpful as patients appear to repeat the recurrent lesions such as VUE, MPVFD/MFI and DVI. Pathologists should be aware of these changes and alert the clinicians to the possibility of recurrences in subsequent pregnancies.

Given the heterogeneity of histologic findings, it would be unlikely that a single treatment would be effective in all cases. Perhaps placental pathology should be used to guide therapy; however, it needs to be validated by a study on a larger sample. Placental examination should be indicated in defined subset of patients which includes maternal autoimmune disorders. 


\section{ACKNOWLEDGMENTS}

Authors would like to thank Dr. Ammu Sivaraman, Emeritus Professor, Department of Pathology, PSGIMSR.

\section{Funding: No funding sources}

Conflict of interest: None declared

Ethical approval: The study was approved by the Institutional Ethics Committee

\section{REFERENCES}

1. Park AL. Placental pathology in antiphospholipid syndrome, In: Hughes' Syndrome, Khamashta MA. Springer-Verlag, London. 2006;362-74.

2. Gómez-Puerta JA. Diagnosis and classification of the antiphospholipid syndrome. J Autoimmunity. 2014;48-49:20-25.

3. Aringer M, Dorner T. Toward new criteria for systemic lupus erythematosus-a standpoint. Lupus. 2016;25:805-11.

4. Perepu U, Rosenstein L. Maternal thrombocytopenia in pregnancy. Obstetr Gynecol. 2013;3(1):6.

5. Rebecca N. Baergen. Umbilical Cord Pathology. Surg pathol. 2013;6:61-85.

6. Raymond W. Redline. Fetal Thrombotic Vasculopathy. Pathology Case Reviews. 2010;15: 37-9.

7. Saleemuddin A, Tanbirojn P, Sirios K. Obstetric and Perinatal Complications in Placentas with Fetal Thrombotic Vasculopathy. Pediatric and Developmental Pathology. 2010;13:459-64.

8. Baergen RN. Manual of Pathology of the Human Placenta, New York, Springer; 2011.

9. Benirschke K, Burton GJ, Baergen RN. Pathology of the Human Placenta, Sixth Edition, New York, Springer-Verlag; 2012.
10. Redline RW. The Placenta. Aliya N. Husain, J. Thomas Stocker, and Louis P. Dehner. Stocker and Dehner's: Pediatric Pathology, 4 th Edition. Wolters Kluwer;2016:325-350.

11. Rodger MA, Walker MC. Is thrombophilia associated with placenta-mediated pregnancy complications? A prospective cohort study. Journal of thrombosis and Haemostasis. 2014;12:469-78.

12. Vijayalakshmi B, Kittali S. Morphological Changes of Placenta in Cases of Pre-eclampsia and Perinatal Outcome. Internat J Scient Study. 2015;3(5):137-42.

13. Redline RW. Distal villous immaturity. MiniSymposium: Placental and Trophoblastic pathology. Diagnost Histopathol. 2012;18(5):189-94.

14. Bagby C, Raymond W. Redline. Multifocal Chorangiomatosis. Pediatr Development Pathol. 2011;14:38-44.

15. McKay EM. Placental diagnoses-placental abruption. In: Amy Heerema-McKenney, Edwina J. Popek and Monique E. De Paepe. eds. Diagnostic Pathology: Placenta, first edition. AMIRSYS publishing, part II. 2015;7-2.

16. Chan JS. Villitis of unknown etiology and massive chronic intervillositis. Surgical pathology clinics. 2013;6(1):115-26.

17. Romero R, Whitten A. Maternal Floor Infarction/Massive Perivillous Fibrin Deposition: A Manifestation of Maternal Antifetal Rejection. Am J Reprod Immunol. 2013;70(4):285-98.

Cite this article as: Ramya $\mathrm{T}$, Umamaheswari $\mathrm{G}$, Chaitra V. Placental pathology in maternal autoimmune diseases-new insights and clinical implications. Int J Reprod Contracept Obstet Gynecol 2017;6:4090-7. 